\title{
Ontologias e Web Services aplicados ao desenvolvimento de Sistemas de Informação Geográfica Móveis Sensíveis ao Contexto
}

\author{
Anderson Resende Lamas, Jugurta Lisboa Filho, \\ Ronoel Matos de Almeida Botelho Júnior, Alcione de Paiva Oliveira \\ Universidade Federal de Viçosa - Departamento de Informática \\ Viçosa, MG, Brasil - CEP: 36570-000 \\ \{andersonlamas, jugurta\}@ufv.br, \{ronoeljr, alcione\} @dpi.ufv.br
}

\begin{abstract}
This article presents the proposal of an architecture aiming at the development of Mobile Geographic Information Systems (Mobile GIS) capable to manage context information. This architecture is based on the specification of a context model based on ontologies and a set of Web Services to access the information stored remotely in a geographic database. This mechanism allows Mobile GIS user to receive personalized information in his mobile device, combining the information of his profile with the exhibition of geo-spatial data.
\end{abstract}

Resumo. Este artigo apresenta a proposta de uma arquitetura para o desenvolvimento de Sistemas de Informação Geográfica Móveis (SIG Móveis) capazes de gerenciar informações de contexto. Esta arquitetura baseia-se na especificação de um modelo de contexto baseado em ontologias e em um conjunto de Web Services para acesso a informações armazenadas remotamente em um banco de dados geográficos. Com este mecanismo é possível que um usuário de um SIG Móvel possa receber informações personalizadas em seu dispositivo móvel, combinando as informações de seu perfil com a exibição de dados geo-espacias.

\section{Introdução}

Vive-se hoje em um período de avanços sem precedentes em tecnologias que exploram a mobilidade e a comunicação entre usuários e dispositivos. Este fato é evidenciado pela constante expansão das infra-estruturas de rede sem fio e a grande proliferação de dispositivos móveis tais como PDA`s (Personal Digital Assistent), Smartphones e telefones celulares. Este cenário tem causado um grande impacto nas mais diversas áreas do conhecimento, especialmente pela possibilidade do desenvolvimento de novas aplicações que exploram tais características.

Na área de Sistemas de Informação Geográfica (SIG), por exemplo, a interseção de dados geo-espacias com dispositivos móveis deu origem a área chamada de SIG Móvel (Mobile GIS). Segundo [Solyman, 2005], SIG Móvel pode ser definido como uma estrutura que integra hardware e software para acesso a dados espaciais (mapas) através de uma rede sem-fio, utilizando-se dispositivos móveis.

Outra área de aplicação que vem crescendo com estes avanços é a de aplicações sensíveis ao contexto (context-aware applications). Estas aplicações são caracterizadas 
por utilizarem informações de contexto para fornecerem serviços e informações relevantes ao usuário durante a realização de alguma tarefa [Dey, 2000]. Neste cenário, contexto pode ser definido como qualquer informação que possa ser utilizada para caracterizar uma pessoa, um lugar ou um objeto, relevantes em uma interação entre um usuário e uma aplicação [Dey, 2000]. Como informações deste tipo têm-se, por exemplo, a identificação e preferências do usuário, sua localização ou características dos dispositivos utilizados.

A integração de informações de contexto em um ambiente de SIG Móvel é um fator que pode agregar um grande valor às informações exibidas para o usuário de uma aplicação. Esta afirmação é possível graças a possibilidade de entrega de informações personalizadas ao usuário, combinadas com a exibição de dados geo-espaciais. Diante deste cenário, este artigo apresenta as características e soluções existentes tanto na área de SIG Móvel quanto de aplicações sensíveis ao contexto e propõe uma arquitetura para o desenvolvimento de aplicações de SIG Móveis capazes de gerenciar informações de contexto.

Na Seção 2 são descritos alguns aspectos relativos à modelagem de contexto. A Seção 3 descreve alguns trabalhos correlatos. Na Seção 4 é apresentada a arquitetura proposta para o desenvolvimento de SIG Móveis sensíveis ao contexto. A Seção 5 apresenta um estudo de caso construído para validar esta arquitetura. Por fim, na Seção 6 são apresentadas as conclusões sobre este trabalho.

\section{Modelagem de Contexto}

Um sistema é caracterizado como sensível ao contexto quando utiliza o contexto para fornecer informação e/ou serviços relevantes ao usuário [Dey, 2000]. Segundo Strang e Linnhoff-Popien [2004] a chave para o desenvolvimento de uma aplicação sensível ao contexto é a definição de um modelo de contexto apropriado, o qual pode ser definido através do uso de ontologias.

As características ontológicas de formalidade, semântica explícita e abstração de implementação habilitam sistemas de software não apenas a inferir novas informações a partir de informações modeladas por ontologias, mas também a compartilhar entre si essas informações de maneira a integrar de forma transparente os serviços que as manipulam [Bulcão Neto, 2006].

Baseado nestas características Bulcão Neto [2006] propõe o modelo SeCoM (Semantic Context Model), um modelo ontológico independente de domínio, descrito na linguagem OWL (Web Ontology Language), que serve como base para a definição de informações de contexto. Este modelo é composto de um conjunto de ontologias interrelacionadas baseadas nas dimensões semânticas de identidade, localização, tempo, atividade e modo de captura e acesso.

SeCoM foi desenvolvido utilizando conceitos genéricos de tal forma que possa ser reutilizado por uma variedade de aplicações sensíveis ao contexto. Para que isto seja possível, as ontologias seguem uma abordagem em duas camadas: a camada superior de ontologias, apresentada na Figura 1, que representa o modelo em si e a camada inferior de ontologias, que é construída com conceitos específicos da aplicação sensível ao contexto que está sendo desenvolvida. Nesta camada, o modelo SeCoM pode ser 
reusado ou mesmo estendido com os conhecimentos que são particulares do domínio da aplicação.

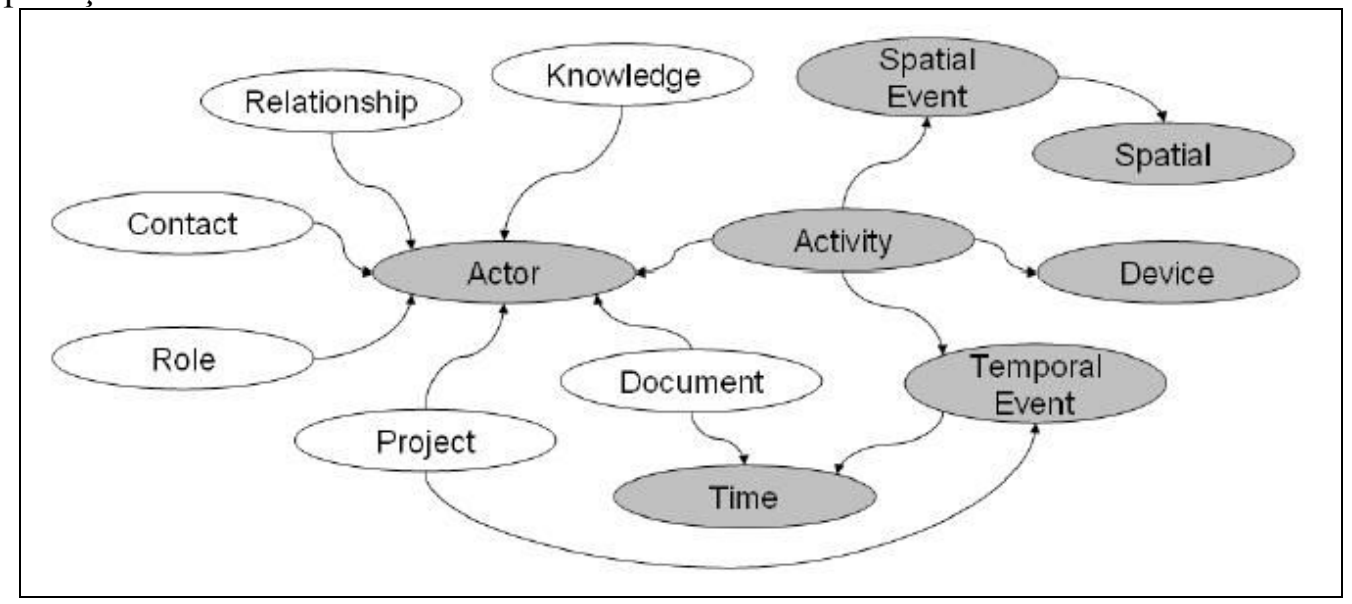

Figura 1 - Modelo SeCoM [Bulcão Neto, 2006].

Com relação ao papel desempenhado pelas ontologias apresentadas na Figura 1, verifica-se as ontologias de apoio Knowledge, Relationship, Role, Contact, Document e Project, as quais modelam diversos aspectos relacionados a um ator (Actor). Neste modelo atores são entidades que executam alguma ação em uma interação usuáriocomputador. Já as ontologias Spatial, Time, Activity e Device modelam, respectivamente, informações de contexto de localização, tempo, atividade e dispositivos computacionais de captura e acesso. As ontologias Spatial Event e Temporal Event são extensões das ontologias Spatial e Time para representar eventos que contenham componentes espaciais e temporais, concomitantemente.

\section{Trabalhos Correlatos}

O desenvolvimento de aplicações de SIG Móveis sensíveis ao contexto vem sendo pesquisado por diversos grupos. A seguir são descritos alguns dos trabalhos que contribuíram para se chegar à arquitetura aqui proposta.

Li et al. [2006] especificam um mecanismo baseado em Web Services para exibir informações contextuais a usuários de dispositivos móveis. As informações contextuais são definidas através da localização do usuário, via GPS, das informações do hardware e das informações dos softwares utilizados. Esta definição de contexto não considera informações do perfil do usuário ou mesmo trata dos dados geo-espaciais.

Weibenberg et. al [2006] descreve a plataforma FLAME2008, desenvolvida para ser utilizada nas Olimpíadas de Pequim. Esta plataforma se caracteriza como uma solução para entrega de informações personalizadas a usuários de dispositivos móveis. Este trabalho utiliza um conjunto de ontologias para definir a situação do usuário, que se caracteriza por tentar inferir as atividades que usuários possam realizar durante um intervalo de tempo. A partir da definição da situação do usuário são exibidas, via $W e b$ Services, as informações que sejam mais adequadas para aquele momento. Apesar de ser um sistema bastante amplo, este trabalho não descreve as implicações de se integrar as informações de contexto em um ambiente de SIG Móvel. 
Pode-se citar ainda o desenvolvimento da arquitetura CoMPASS (Combining Mobile Personalized Applications with Spatial Services) [Weakliam et. al, 2005] a qual utiliza, além das informações de perfil e localização, a trajetória do usuário para automaticamente entregar informações personalizadas a um cliente de SIG Móvel. A definição e o armazenamento das informações de contexto baseiam-se na utilização de um conjunto de arquivos de log. Esta abordagem difere da solução proposta neste artigo, a qual sugere o uso de ontologias para definição das informações de contexto e o uso de um banco de dados geográficos para armazenamento dos dados.

\section{A Arquitetura Context-Mobile-GIS}

A arquitetura proposta neste trabalho, batizada de Context-Mobile-GIS (CM-GIS), tem como objetivo a construção de SIG Móveis integrados a aplicações sensíveis ao contexto.

No caso dos SIG Móveis, conforme definido por Tomko [2003], o desenvolvimento de aplicações requer atenção aos seguintes elementos: (a) armazenamento e consulta a dados em um banco de dados geográficos; (b) prover um mecanismo para realização de consultas e acesso a dados localizados remotamente; (c) a correta utilização de tecnologias.

Com relação ao banco de dados geográficos (a) deve-se utilizar algum Sistema de Gerenciamento de Banco de Dados (SGBD) que tenha capacidade para armazenar e gerenciar dados geo-espacias. Alguns SGBD disponíveis atualmente disponibilizam esta funcionalidade através de uma extensão para dados espaciais, como é o caso do Oracle, do SQLServer, do PostGreSQL, dentre outros. Para tratar do acesso remoto às informações (b), uma solução bastante adotada é o uso de Web Services, devido a sua capacidade de disponibilizar serviços que podem ser acessados dinamicamente através de uma rede. Por fim, com relação às tecnologias adotadas (c), é importante que se escolha uma ferramenta capaz de manipular e exibir dados geo-espaciais, tanto em sua forma gráfica quanto descritiva, nos dispositivos móveis.

Já no caso das aplicações sensíveis ao contexto é importante que se defina um mecanismo capaz de representar e manipular o contexto envolvido no domínio que está sendo tratado pela aplicação. Como o modelo SeCoM foi definido de forma genérica, no presente trabalho sugere-se a sua utilização na definição do modelo de contexto.

Diante das considerações feitas foi proposta a arquitetura CM-GIS para o desenvolvimento de aplicações de SIG Móveis sensíveis ao contexto, conforme apresentado na Figura 2. Esta arquitetura prevê que para o desenvolvimento de aplicações de SIG Móveis capazes de gerenciar informações de contexto são necessários os seguintes elementos:

(1) a aplicação de SIG Móvel, a qual será a interface para o cliente a partir de seu dispositivo móvel. Um requisito importante para esta aplicação é que ela tenha capacidade de manipular e exibir dados geo-espaciais;

(2) um conjunto de Web Services para gerenciar a comunicação e o fornecimento de serviços e informações ao usuário. A utilização de Web Services tem uma grande importância nesta arquitetura, ao permitir que operações que demandem uma alta carga 
de processamento possam ser realizadas em máquinas mais robustas e não diretamente no dispositivo móvel;

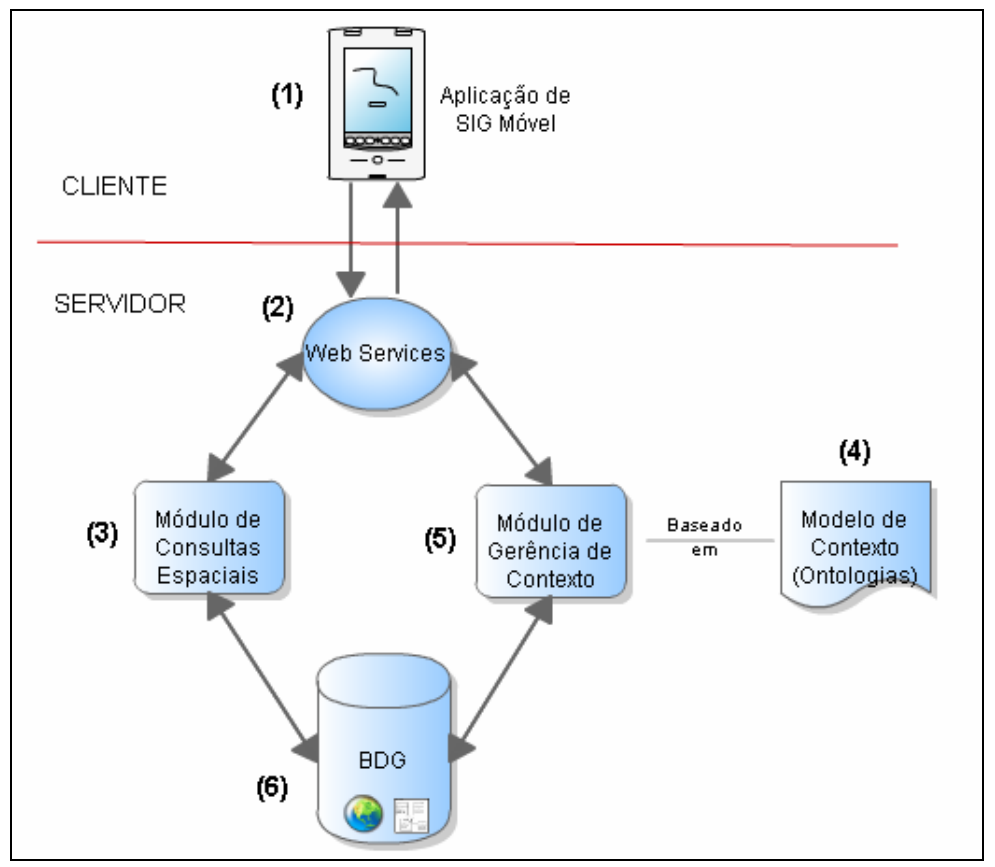

Figura 2 - Arquitetura Context-Mobile-GIS (CM-GIS)

(3) um módulo (Módulo de Consultas Espaciais) que se comunica com os Web Services e realiza consultas espaciais, através da linguagem SQL, sobre as informações armazenadas no banco de dados geográficos;

(4) um conjunto de ontologias que servem para modelar o contexto envolvido na aplicação. É sugerido para a modelagem do contexto o uso do modelo SeCoM apresentado na Seção 2;

(5) um módulo (Módulo de Gerência de Contexto) definido a partir do modelo de contexto baseado em ontologias que se comunica com os Web Services para fornecer informações de contexto. As informações de contexto também devem estar armazenadas no banco de dados e consultadas via SQL; e

(6) um banco de dados geográficos que gerencia todos os dados geo-espaciais e descritivos envolvidos na aplicação.

Estes itens devem ser detalhados conforme a aplicação que será desenvolvida. $\mathrm{Na}$ próxima Seção é apresentado um estudo de caso, construído com base nesta arquitetura.

\section{UFV-GeoMobile: um estudo de caso}

Nesta Seção é descrito o desenvolvimento do sistema UFV-GeoMobile. Este sistema teve como alvo o campus da Universidade Federal de Viçosa (UFV), que é uma instituição pública de ensino superior, cujo principal campus está localizado na cidade de Viçosa, MG. A UFV oferece diversos cursos de graduação e pós-graduação, em 
diversas áreas do conhecimento. Anualmente recebe um grande volume de pessoas que visitam ou que estabelecem algum vínculo com o seu campus. Dentre estas pessoas pode-se citar os novos estudantes ( 2000 por ano), participantes de congressos, pesquisadores visitantes, ou simplesmente pessoas que desejam visitar suas dependências. Além disso, existem também as pessoas que já possuem alguma ligação com a Universidade, tais como professores, servidores ou ainda os estudantes egressos. Um ponto em comum entre estes diversos indivíduos é que muitas vezes eles têm dificuldades em identificar lugares ou serviços no campus, dada sua grande extensão territorial e à velocidade com que surgem novas construções e instalações diversas.

Considerando este cenário, verifica-se que o mesmo é ideal para a implementação de um sistema que valide a arquitetura CM-GIS, proposta neste trabalho. Seria interessante que houvesse uma fonte prática e automatizada para o usuário buscar informações sobre o campus. Seria ainda mais adequado que as opções de consulta e a obtenção das informações fossem personalizadas de acordo com o contexto em que o usuário se encontra. Como exemplo, pode-se citar que um visitante provavelmente não está interessado no horário da próxima aula, mas gostaria de informações sobre pontos de serviço (banco, farmácia, etc) ou mesmo de se informar sobre o local de realização de algum evento que ele esteja interessado.

Diante desta realidade foi implementado o sistema UFV-GeoMobile, o qual permite que um usuário utilizando uma aplicação de SIG Móvel instalada em um PDA possa realizar consultas ou mesmo receber informações personalizadas sobre o campus da UFV. Esta aplicação tem como base um mapa do campus devidamente gerenciado por um componente para manipulação de dados geográficos. Além disso, possui um conjunto de telas com as opções de consulta e um mecanismo para exibição de informações baseadas no contexto do usuário. Mais especificamente a definição de contexto baseia-se em uma agenda de atividades a serem realizadas pelo usuário para assim exibir a localização geográfica do local onde será realizada esta atividade.

Todo o processo de desenvolvimento é descrito nas seções que se seguem.

\subsection{O modelo de contexto: ontologia de um campus universitário}

O modelo de contexto para o estudo de caso UFV-GeoMobile deve conter informações sobre as estruturas e as atividades realizadas em um campus universitário. Dentre as informações que devem ser modeladas para refletir este domínio estão:

- As pessoas: neste caso envolvem estudantes, funcionários e visitantes. Os funcionários podem ser professores ou servidores técnico-administrativos;

- A estrutura administrativa: reflete a organização hierárquica de uma universidade, incluindo reitoria, centros de ensino, departamentos e órgãos;

- A estrutura acadêmica: envolve atividades relacionadas ao ensino, pesquisa e extensão;

- Agenda de atividades: corresponde ao conjunto de tarefas que uma pessoa possa realizar no campus. Este item é fundamental para o sistema resolver informações de contexto; 
- Dados geo-espaciais: utilizado para representar espacialmente os elementos que possuem localização geográfica no campus. Exemplos destes elementos são as construções e os serviços instalados em cada uma destas construções.

A partir destas informações parte-se para uma representação formal de um modelo de contexto. O modelo de contexto do sistema UFV-GeoMobile foi feito com base no modelo SeCoM, proposto por Bulcão Neto [2006], do qual foram aproveitados os conceitos ontolológicos de Actor, Activity, Spatial Event e Temporal Event.

Este modelo de contexto tem grande importância no desenvolvimento da aplicação, pois serviu de base para a modelagem do Banco de Dados Geográfico e para a implementação das funcionalidades disponíveis no módulo de gerência de contexto. Por limitações de espaço, a representação formal do modelo de contexto não é apresentada neste trabalho.

\subsection{Construção do banco de dados geográficos}

O Banco de Dados Geográficos do sistema UFV-GeoMobile foi construído a partir da junção de um conjunto de dados geográficos, mantidos pela administração da UFV, associados a um conjunto de bases relacionais utilizadas por uma variedade de sistemas administrativos, também mantidos pela instituição. Os dados geográficos foram alterados para se adequarem aos formatos de dados manipulados pelo aplicativo de SIG Móvel. Inicialmente os dados estavam armazenados no formato do sistema AutoCad e foram convertidos para o formato Shapefile, utilizando-se o programa cad2shape [Cad2Shp, 2006]. As seguintes camadas foram produzidas: logradouros, construções e instalações específicas dentro de uma construção, como órgãos administrativos e laboratórios.

A modelagem conceitual do banco de dados, conforme visualizado na Figura 3, foi definida a partir das informações modeladas na ontologia de domínio de um campus universitário. Para esta tarefa foi utilizado o modelo UML-GeoFrame [Lisboa Filho \& Iochpe, 2008], que é um modelo específico para bancos de dados geográficos. Posteriormente, o modelo conceitual foi implementado utilizando-se o SGBD PostGreSQL com sua extensão espacial PostGIS.

No diagrama da Figura 3 pode-se observar que o sistema possui classes com e sem representação espacial. De acordo com o modelo UML-GeoFrame, as classes e subclasses Funcionário, Professor, Técnico Administrativo, Pessoa, Estudante, Visitante, Agenda, Atividade, Órgão e Tipo de Instalação são especializações de ObjetoNãoGeográfico $(\triangle)$, ou seja, não possuem representação espacial. As classes Construção, Logradouros e Instalação são percebidas na visão de objetos $(\triangle)$ e possuem representação espacial do tipo Área, Linha e Ponto, respectivamente. Por fim, as classes ImagemAérea e CurvaDeNivel são percebidas na visão de campo $(\bullet)$. A primeira possui representação espacial do tipo GradeDeCélulas (曲), enquanto a segunda possui representação do tipo Isolinhas (图).

\subsection{A aplicação SIG Móvel}

A interface gráfica do SIG Móvel foi desenvolvida utilizando o framework Microsoft ${ }^{\circledR}$ Visual Studio 2005, a linguagem C\# .NET e o emulador Pocket PC 2003. Para a visualização dos dados geo-espacias foi utilizado o software SharpMap, o qual consiste em um conjunto de controles baseados na plataforma .NET para construção de 
aplicativos de SIG em dispositivos móveis [SharpMap, 2008]. Uma versão inicial deste protótipo foi publicada em [Lamas et al., 2008].

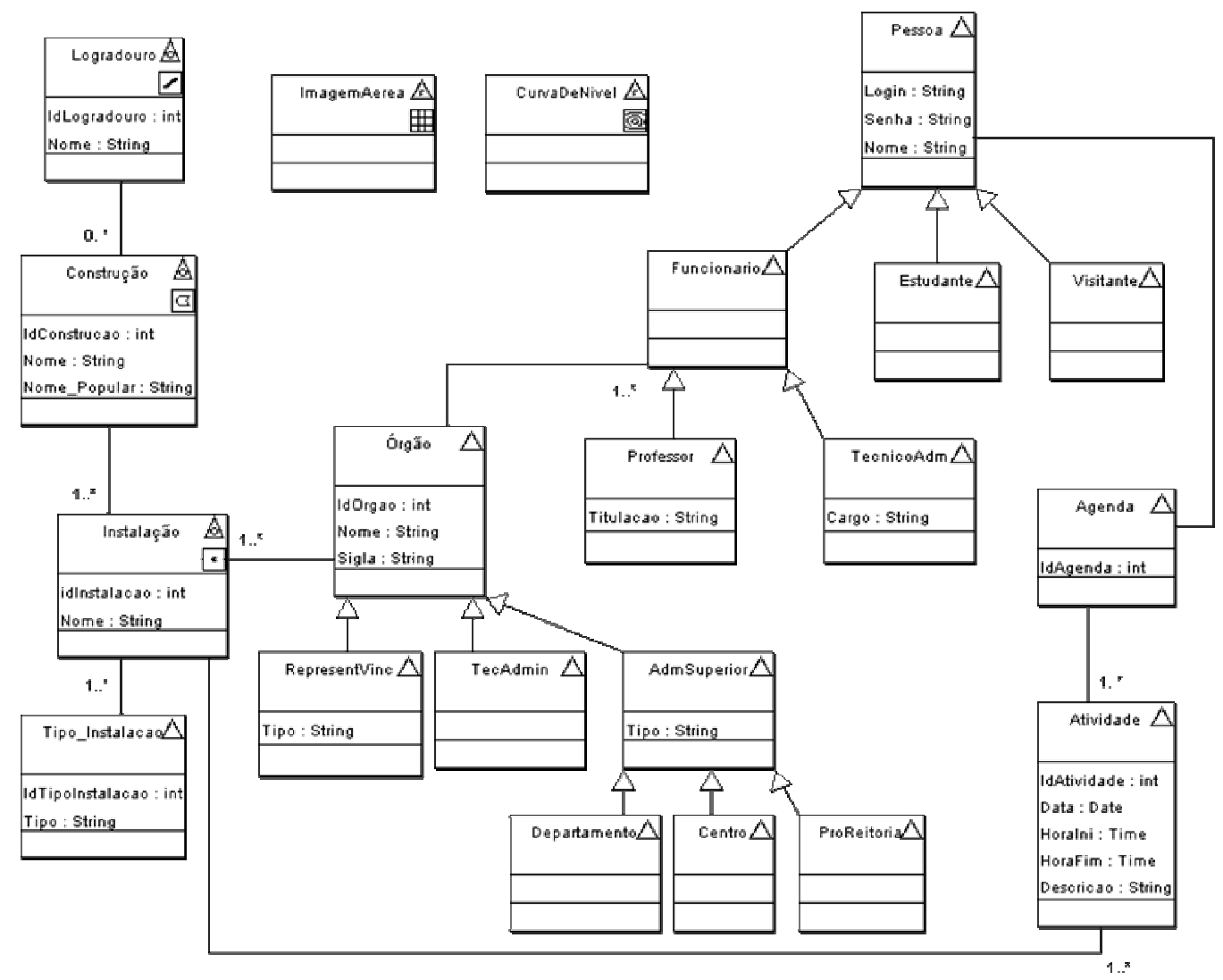

Figura 3 - Modelagem do Banco de Dados Geográfico para o UFV-GeoMobile

Para permitir o acesso as informações armazenadas no banco de dados geográfico foi desenvolvido um Web Service na linguagem Java (SOAP), utilizando-se a IDE NetBeans 6.1 e o servidor GlassFish. Para cada funcionalidade disponibilizada no aplicativo de SIG Móvel foi desenvolvida uma operação correspondente no Web Service, para receber os parâmetros e repassá-los ao Módulo de Consultas Espaciais ou ao Módulo de Gerência de Contexto. Para exemplificar estas operações são descritas neste trabalho a opção de localizar uma instalação ou serviço e o mecanismo que informa ao usuário sobre a localização geográfica de uma atividade a ser realizada por ele.

Para a localização de um serviço ou instalação, inicialmente o usuário digita o nome completo ou parcial do item desejado, utilizando a tela apresentada na Figura 4-a. Ao digitar este nome e clicar no botão "Pesquisar" é invocada a operação "getLocal", descrita em detalhes no Quadro 1. Esta operação recebe como parâmetros o nome digitado e as coordenadas ( $\mathrm{x}, \mathrm{y})$ do usuário, obtidas via GPS ou via um mecanismo implementado no SIG Móvel que permite ao próprio usuário indicar sua localização, inserindo um ícone no mapa. Esta segunda opção foi implementada para que o usuário possa utilizar o aplicativo UFV-GeoMobile, mesmo que o PDA não possua um GPS embutido. 
A operação "getLocal" repassa seus parâmetros para o módulo de consulta, o qual gera uma consulta SQL, também descrita no Quadro1, que retorna os nomes das instalações que correspondem ao nome digitado, a distância de cada instalação em relação a posição do usuário e as coordenadas do ponto central da edificação na qual a instalação esteja alocada. A seleção feita pela consulta SQL é retornada para o dispositivo móvel, que exibe uma lista das instalações disponíveis (Figura 4-a). Ao selecionar um dos itens desta lista, a aplicação indica, através de um pequeno ícone desenhado sobre o mapa, a localização geográfica da instalação escolhida, conforme visualizado na Figura 4-b.

O mecanismo para notificar o usuário do SIG Móvel sobre a localização geográfica de alguma atividade que esteja cadastrada em sua agenda, foi desenvolvido como uma opção sensível ao contexto do usuário. Inicialmente para ter acesso a esta funcionalidade o usuário deve se identificar no sistema através de um login e uma senha. A partir deste momento, de minuto em minuto é invocada operação "GetEvento", apresentada no Quadro 2. Esta operação recebe como parâmetro além do login e da senha, a hora atual, que é obtida do dispositivo móvel. Esta operação repassa estes parâmetros para o módulo de gerência de contexto o qual gera uma consulta em SQL, também apresentada no Quadro 2. Esta consulta retorna todas as atividades cadastradas do usuário que faltem 10 minutos para seu início, detalhando a descrição da atividade, o horário de início e de término desta atividade, o nome da instalação na qual a atividade irá ser realizada e as coordenadas do ponto central da edificação na qual a instalação está alocada. A partir destas informações, a aplicação de SIG Móvel exibe uma caixa de texto para o usuário e indica através de um ícone a localização geográfica desta atividade, conforme observado na Figura 5.

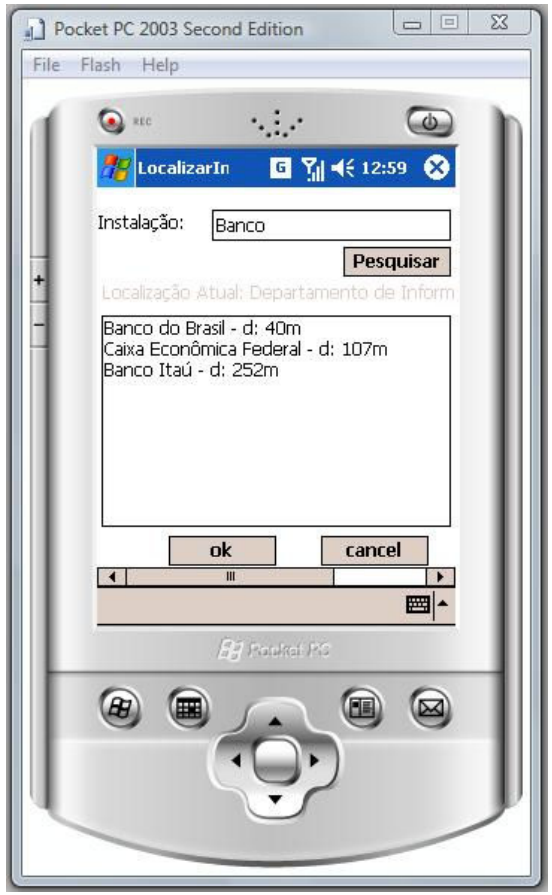

(a) Consulta instalações

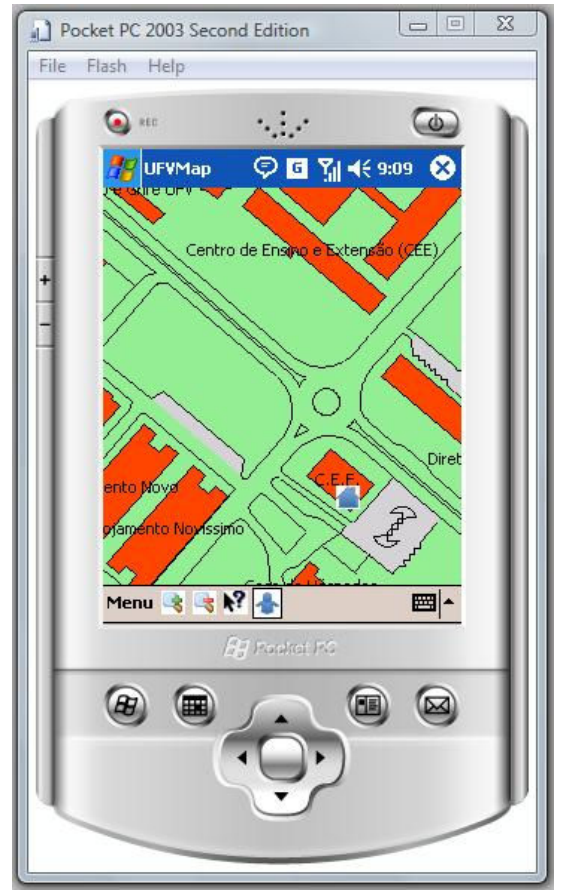

(b) Localização de uma instalação

Figura 4 - Telas de consulta a instalações no sistema UFV-GeoMobile 
Quadro 1 - Operação do Web Service e consulta SQL para localizar instalações.

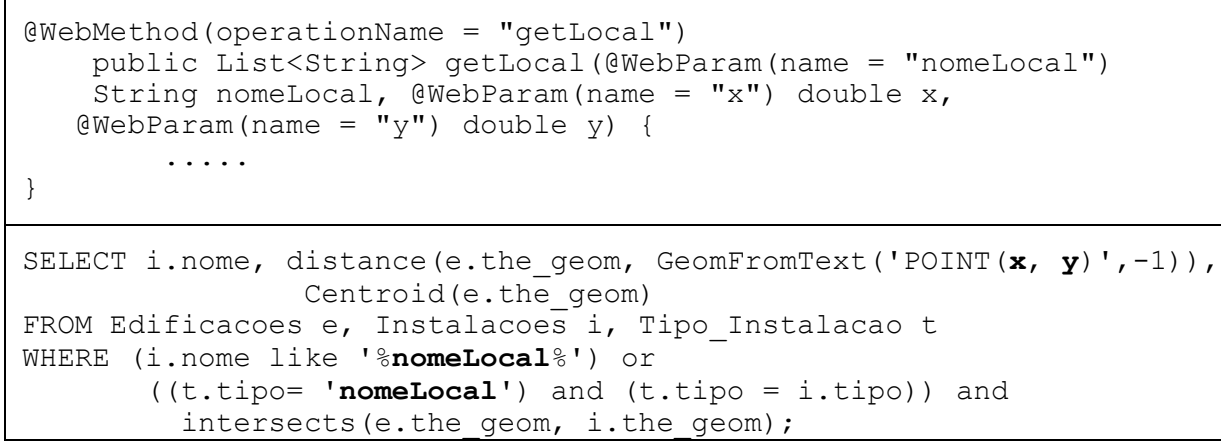

\section{Quadro 2 - Operação do Web Service e consulta SQL para avisar sobre eventos.}
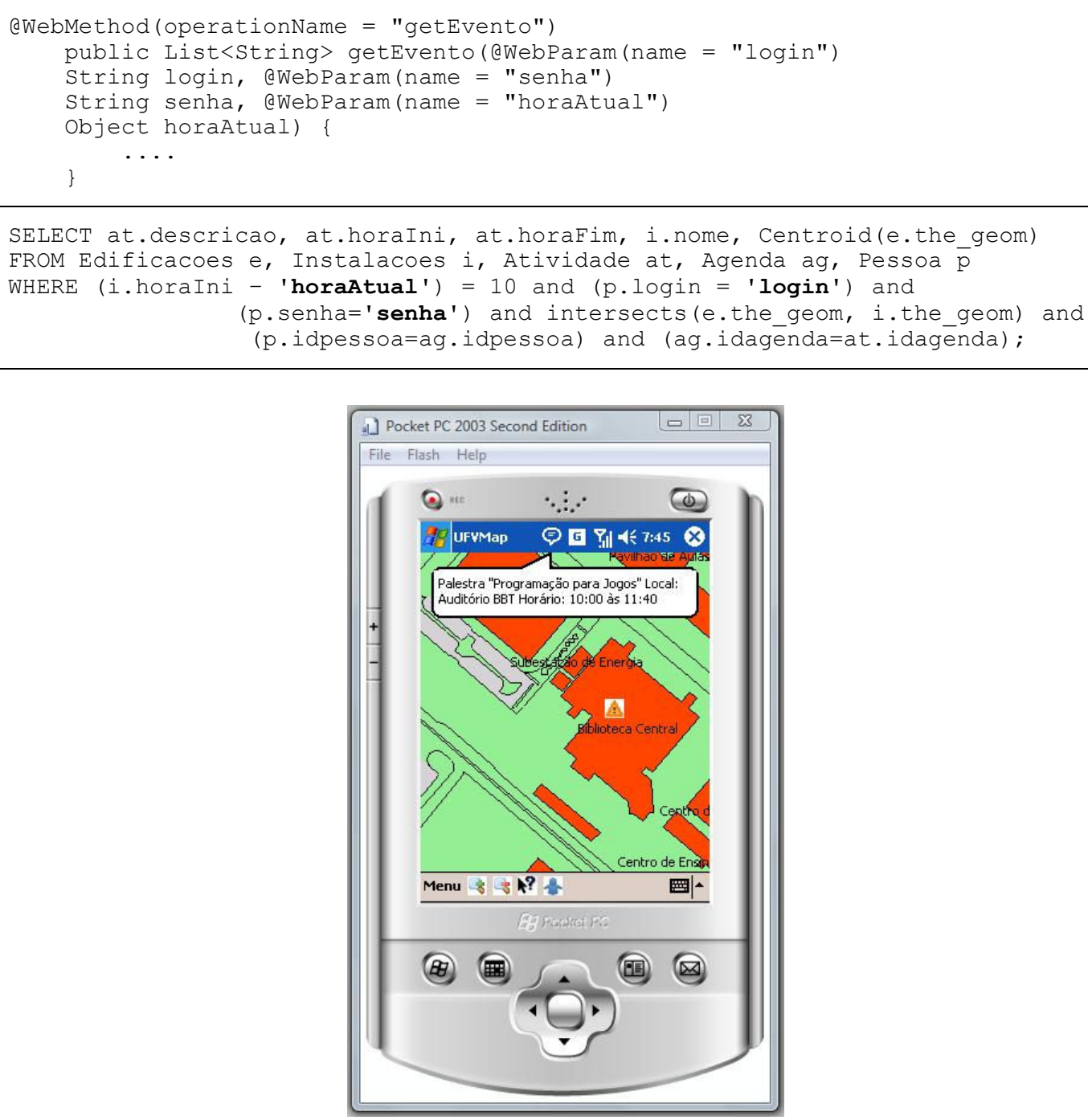

Figura 5 - Tela que notifica o usuário sobre a realização de alguma atividade. 


\section{Conclusões}

Neste trabalho foi apresentada a especificação de uma arquitetura para o desenvolvimento de SIG Móveis capazes de gerenciar informações de contexto. Foram estudadas as características inerentes a este tipo de aplicação até que se chegasse a solução proposta, a qual inclui: o uso de Web Services para permitir o acesso remoto a informações a partir do dispositivo móvel, ontologias para a modelagem de contexto e o uso de banco de dados geográficos para o armazenamento das informações.

O uso de ontologias para especificar um modelo de contexto tem como principal vantagem a possibilidade de especificar o correto significado e relacionamento entre os termos, evitando interpretações imprecisas sobre o domínio que está sendo modelado. A adoção do modelo SeCoM pode facilitar bastante este processo de modelagem, visto que ele aborda as diversas dimensões de informação contextual e foi desenvolvido com característica de independência de domínio.

O uso de ontologias neste trabalho poderia ser melhor explorado se houvesse um mecanismo que manipulasse as ontologias e inferisse novas informações antes de retorná-las ao usuário do SIG Móvel. Esta abordagem será feita em um trabalho futuro.

Outra característica que pode ser futuramente explorada é o uso de Geo Web Services, os quais são serviços definidos pela OGC (Open GIS Consortium) para tratar especificamente de dados geo-espaciais. Este mecanismo evitaria a necessidade da instalação de um aplicativo no PDA, conforme foi feito na aplicação UFV-GeoMobile. Ao invés disso, o aplicativo de SIG Móvel poderia ser acessado através de um browser.

Contudo, a arquitetura apresentada, juntamente com a estrutura UFV-GeoMobile desenvolvida como estudo de caso, mostram como o uso de um aplicativo de SIG Móvel capaz de gerenciar informações de contexto pode ser de grande utilidade para um usuário, especialmente pela capacidade de apresentar informações personalizadas combinadas com a exibição de dados geo-espaciais. Desse modo, este trabalho apresenta uma forma viável e bastante prática para o desenvolvimento de aplicações de SIG Móvel, apresentando soluções para tratar desafios típicos de aplicações que envolvam dados geo-espaciais, contexto e dispositivos móveis.

\section{Agradecimentos}

Projeto parcialmente apoiado com recursos da FAPEMIG - Fundação de Amparo à Pesquisa do Estado de Minas Gerais e do CNPq/MCT/CT-Info.

\section{Referências}

Bulcão Neto, R. F. (2006). Um processo de software e um modelo ontológico para apoio ao desenvolvimento de aplicações sensíveis ao contexto. São Paulo. Tese (Doutorado em Ciência da Computação) - Universidade de São Paulo.

Cad2Shp (2006). Cad2Shp: AutoCAD DXF/DWG to ArcView Shapefile Converter. Disponível em: <http://www.brightergraphics.co.uk/guthrie/ cad2shp.htm>. Acesso em: 12 de fevereiro de 2006.

Dey, A. K. (2000). Providing architectural support for building context-aware applications. Estados Unidos. PhD thesis (Doutorado em Ciência da Computação) Georgia Institute of Technology. 
Lamas, A. R.; Lisboa Filho, J.; Oliveira, A. P.; Lopes, A. M. (2008). Sistemas de Informação Geográfica Móveis orientados ao contexto: uma abordagem baseada em ontologias de domínios. In: Anais do IV Simpósio Brasileiro de Sistemas de Informação (SBSI 2008). Rio de Janeiro: SBC. pp. 70-81.

Li, X.; Shin, W.; Li, L.; Yoo, S. B. (2006). GIS Web Service Using Context Information in Mobile Environments. In: Proceedings of ICCSA 2006, LNCS 3980, pp. 895 903.

Lisboa Filho, J.; Iochpe, C. (2008). Modeling with a UML profile. In: Shashi Shekhar and Hui Xiong. Encyclopedia of Geographical Information Science. New York: Springer. pp. 691-700.

SharpMap (2008). SharpMap: Geospatial Application Framework for the CLR. Disponível em: $<$ http://www.codeplex.com/SharpMap>. Acesso em: 17 de agosto de 2008.

Solyman, A. A (2005). Investigating Mobile GIS. Disponível em: $<$ http://www.directionsmag.com/article.php/>. Acesso em: 28 de maio de 2007.

Strang, T.; Linnhoff-Popien, C. (2004). A Context Modeling Survey. In: Proceedings of Workshop on Advanced Context Modelling, Reasoning and Management (UbiComp4). Nottingham-England.

Tomko, M. (2003). Spatial Databases for Mobile GIS Applications. Eslováquia. Dissertação (Mestrado em Engenharia Civil) - Slovak University of Technology.

Weakliam, J., et al. (2005). Delivering Personalized Context-Aware Spatial Information to Mobile Devices. In: Proceedings of W2GIS 2005, LNCS 3833, pp. 194-205.

Weißenberg, N.; Gartman, R.; Voisard, A. (2006). An Ontology-based Approach to Personalized Situation-aware Mobile Service Supply. GeoInformatica, v.10, n.1, pp. 55-90. 